\title{
Tetrasubstituted Imidazolium Salts as Potent Antiparasitic Agents against African and American Trypanosomiases
}

\author{
Ouldouz Ghashghaei ${ }^{1}$, Nicola Kielland ${ }^{1}$, Marc Revés ${ }^{1}$, Martin C. Taylor ${ }^{2}$, John M. Kelly ${ }^{2}$, \\ Ornella Di Pietro $^{3}$, Diego Muñoz-Torrero ${ }^{3}$ iD, Belén Pérez ${ }^{4}$ and Rodolfo Lavilla ${ }^{1, *}$ \\ 1 Laboratory of Organic Chemistry, Faculty of Pharmacy and Food Sciences, University of Barcelona, \\ Barcelona Science Park, Baldiri Reixac 10-12, 08028 Barcelona, Spain; ouldouz.ghashghaei@gmail.com (O.G.); \\ nicola.kielland@gmail.com (N.K.); revesmarc@gmail.com (M.R.) \\ 2 Department of Pathogen Molecular Biology, London School of Hygiene and Tropical Medicine, \\ London WC1E 7HT, UK; Martin.Taylor@lshtm.ac.uk (M.C.T.); john.kelly@lshtm.ac.uk (J.M.K.) \\ 3 Laboratory of Pharmaceutical Chemistry (CSIC Associated Unit), Faculty of Pharmacy and Food Sciences, \\ and Institute of Biomedicine (IBUB), University of Barcelona, 08028 Barcelona, Spain; \\ od265@cam.ac.uk (O.D.P.); dmunoztorrero@ub.edu (D.M.-T.) \\ 4 Department of Pharmacology, Therapeutics and Toxicology, Institute of Neurosciences, \\ Autonomous University of Barcelona, 08193 Bellaterra, Barcelona, Spain; belen.perez@uab.cat \\ * Correspondence: rlavilla@ub.edu; Tel.: +34-934037106
}

Received: 29 November 2017; Accepted: 13 January 2018; Published: 16 January 2018

\begin{abstract}
Imidazolium salts are privileged compounds in organic chemistry, and have valuable biological properties. Recent studies show that symmetric imidazolium salts with bulky moieties can display antiparasitic activity against T. cruzi. After developing a facile methodology for the synthesis of tetrasubstituted imidazolium salts from propargylamines and isocyanides, we screened a small library of these adducts against the causative agents of African and American trypanosomiases. These compounds display nanomolar activity against $T$. brucei and low (or sub) micromolar activity against T. cruzi, with excellent selectivity indexes and favorable molecular properties, thereby emerging as promising hits for the treatment of Chagas disease and sleeping sickness.
\end{abstract}

Keywords: imidazolium salts; isocyanides; antiparasitic agents; Trypanosoma brucei; Trypanosoma cruzi

\section{Introduction}

Chagas disease and sleeping sickness are caused by infection with the protozoan parasites Trypanosoma cruzi and Trypanosoma brucei sp., respectively, and have been classified as Neglected Tropical Diseases. Sleeping sickness threatens millions of people in 36 countries in sub-Saharan Africa. Chagas disease is estimated to affect around 8 million people in endemic areas of 21 Latin American countries. The population at risk of these parasitic diseases normally has limited access to medical assistance, as they live in rural areas of developing countries. Moreover, due to immigration, war, and poverty, the control of infection can be complicated. Indeed, in recent years, an increasing number of Chagas disease cases have been reported in the US, European countries, and Canada [1,2].

Both infections are transmitted by insect vectors. In the case of Chagas disease, transmission can also occur by the congenital route, food contamination, blood transfer, and organ transplantation. Pathology in Chagas disease is mainly associated with the chronic stage, which occurs years after the initial infection, with cardiomyopathy being a common outcome. In African trypanosomiasis, second-stage disease-which occurs once parasites breach the blood brain barrier (BBB)-is the critical phase, being fatal unless treated. Due to serious side effects and other limitations of the currently available drugs, there is an urgent need to develop efficient medications against these diseases [3-7]. 
Imidazolium ions are valuable heterocyclic scaffolds due to their broad applications, especially in organocatalysis, and in the synthesis of liquid crystals. Recently, the biological activities of imidazolium and benzimidazolium ions has been reviewed, assessing their toxicity and particularly their wide range of uses in medicinal chemistry [8]. In 2014, Robello et al. [9] reported that simple symmetric imidazolium salts A (Figure 1) with bulky aromatic moieties on the $\mathrm{N}^{1}$ and $\mathrm{N}^{3}$ positions are active against T. cruzi. The putative biological target of these compounds has been postulated as being linked with the parasite mitochondrion. The major differences between these organelles in parasites and mammals could explain the selective toxicity of the imidazolium salts toward trypanosomes $[8,9]$. In this respect, we recently reported the potent antitrypanosomal activity of a family of azine-fused aminoimidazolium salts B (Figure 1) arising from a trimethylsilyl-catalyzed interaction of azines with isocyanides in a multicomponent manner [10]. Analogously, these compounds also presented two diversity points at the $\mathrm{N}$ atoms, and the most active hits displayed aliphatic residues at these positions.

Having developed a modular methodology for the facile synthesis of complex imidazolium salts [11], the goal of the present work was to study the antiparasitic activity of more complex unsymmetrical tetrasubstituted imidazolium salts 1 (Figure 1) against both African and American trypanosomiases. Relevantly, the target compounds feature a novel substitution pattern affecting not only the $\mathrm{N}$ atoms, but also at $\mathrm{C}$ atoms at positions 4 and 5 .

Previous work

by Robello et al. [9]<smiles>[R]n1cc[n+]([O-])c1</smiles>

A

Symmetric imidazolium salts with one/two diversity points Active aganist $T$. cruzi
Previous work

by Kishore et al. [10]<smiles>[R]Nc1c2c3ccccc3cc[n+]2cn1[R]</smiles>

Fused isoquinoline-imidazolium salts with two diversity points Acitve against $T$. cruzi and $T$. brucei
Present work<smiles></smiles>

Non-symmetric imidazolium salts with four diversity points

Figure 1. Structures of imidazolium salts active against Trypanosoma species: disubstituted imidazolium salts A [9], amino isoquinoline-imidazolium salts $\mathbf{B}$ [10], and the novel tetrasubstituted imidazolium salts $\mathbf{1}$.

\section{Results and Discussion}

\subsection{Chemistry}

Compounds 1a-e can be easily accessed through the novel two-step condensation of amines, aldehydes, terminal alkynes, and isocyanides [11]. First, the amine, aldehyde, and terminal alkyne substrates condense through a multicomponent A3 reaction to yield propargyl amines 2 in high yields [12]. In the second step, the formed propargylamine and isocyanide interact through an acid-catalyzed insertion-cyclization and isomerization process to give the desired imidazolium salts 1a-e in good-to-moderate yields (Scheme 1) [11]. Interestingly, this two-step sequence can be performed in tandem. A similar process was reported by Zhu, featuring a dual catalytic system of AgOTf and $\mathrm{Yb}(\mathrm{OTf})_{3}$ [13]. The nature, scope, and commercial availability of the starting materials, the mild reaction conditions, and the modular access to imidazolium salts 1 (Table 1), with four diversity points, makes this synthetic approach ideal for medicinal chemistry, especially for hit finding through screening. In this respect, is relevant to comment that restrictions on the involved chemistry and the practical access to the alkyne inputs guided the initial screen on the substituent range at this initial stage. The A3 reaction with primary amines works better with anilines and aromatic aldehydes, and for these reason, $R^{1}$ and $R^{2}$ are substituted phenyl rings. On the other hand, there is a limited choice of commercially available alkynes; the most suitable/cheap are arylacetylenes $\left(R^{3}\right.$ being also phenyl or $p$-Me-phenyl), whereas there is a wider scope on $\mathrm{R}^{4}$ as many isocyanides (aliphatic, aromatic, functionalized) conveniently react in the second step of the sequence [11]. 


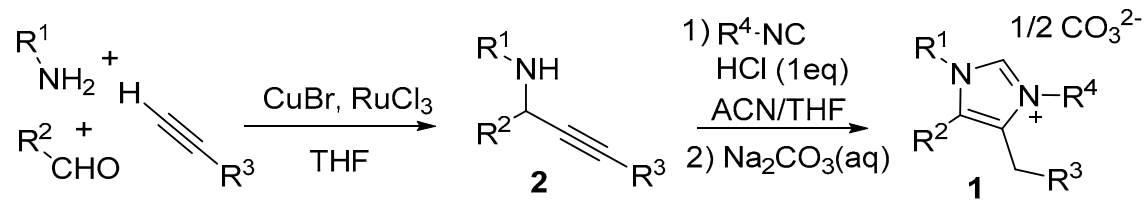

Scheme 1. Synthetic access to the imidazolium salts 1. ACN: Acetonitrile.

Table 1. Structural description of imidazolium salts 1.

\begin{tabular}{|c|c|c|c|c|c|}
\hline Compd. & $\mathbf{R}^{1}$ & $\mathbf{R}^{2}$ & $\mathbf{R}^{3}$ & $\mathbf{R}^{4}$ & $1 / 2 \mathrm{CO}_{3}^{2-}$ \\
\hline $1 \mathrm{a}$ & $p$-Me-Ph & $\mathrm{Ph}$ & $\mathrm{Ph}$ & tert-Bu & \\
\hline $1 b$ & $p$-MeO-Ph & $p$-Me-Ph & $\mathrm{Ph}$ & $p$-MeO-Ph & \\
\hline 1c & $p$-MeO-Ph & $p$-Cl-Ph & $p$-Me-Ph & tert-Bu & \\
\hline 1d & $p-\mathrm{Me}-\mathrm{Ph}$ & $p$-Cl-Ph & $\mathrm{Ph}$ & 2-naphthyl & \\
\hline 1e & $p$-Me-Ph & $\mathrm{Ph}$ & $\mathrm{Ph}$ & $-\mathrm{COOH}$ & 1 \\
\hline
\end{tabular}

\subsection{Biological Activity against T. brucei and T. cruzi}

Next, we evaluated the trypanocidal activity of salts $\mathbf{1}$ against bloodstream forms of T. brucei and the epimastigote form of T. cruzi (Materials and Methods) (Table 2). Compounds 1a-d displayed a potent bioactivity profile at low or sub-micromolar (T. cruzi) or nanomolar levels (T. brucei). For comparison, the $\mathrm{EC}_{50}$ value for nifurtimox - a drug used to treat T. brucei and T. cruzi infections-is approximately $2 \mu \mathrm{M}$ for both parasites [14]. In contrast to compounds 1a-d, salt 1e was significantly less active, possibly due to its zwitterion character in physiological conditions, which could compromise its ability to cross biological membranes. Compounds $\mathbf{1} \mathbf{b}$ and $\mathbf{1 d}$ exhibited the highest potency against both parasites, being clearly more active against $T$. brucei. Interestingly, both compounds feature aromatic moieties on $\mathrm{N}^{1}$ and $\mathrm{N}^{3}$. The cytotoxicity of these compounds was evaluated using L6 rat myoblast cells (Materials and Methods). They displayed high selectivity indexes, particularly with T. brucei, suggesting good potential for use as antitrypanosomal drugs.

Table 2. Biological activity of compounds 1 vs. bloodstream form T. brucei and T. cruzi epimastigotes.

\begin{tabular}{|c|c|c|c|c|c|}
\hline & \multirow{2}{*}{$1 \mathbf{a}$} & \multirow{2}{*}{$1 b$} & \multirow{2}{*}{ 1c } & \multirow{2}{*}{$1 d$} & \multirow{2}{*}{$1 e$} \\
\hline & & & & & \\
\hline $\mathbf{E C}_{50}{ }^{1}$ (T. brucei, $\left.\mu \mathrm{M}\right)$ & $0.18 \pm 0.01$ & $0.04 \pm 0.00$ & $0.18 \pm 0.01$ & $0.07 \pm 0.00$ & $5.26 \pm 0.01$ \\
\hline $\mathbf{E C}_{90}{ }^{2}$ (T. brucei, $\left.\mu \mathrm{M}\right)$ & $0.28 \pm 0.07$ & $0.05 \pm 0.00$ & $0.20 \pm 0.01$ & $0.09 \pm 0.00$ & $6.79 \pm 0.09$ \\
\hline $\mathrm{EC}_{50}(\mathrm{~L} 6$ cells, $\mu \mathrm{M})$ & $33.90 \pm 3.30$ & $4.60 \pm 0.38$ & $9.69 \pm 0.58$ & $4.09 \pm 0.28$ & $>130$ \\
\hline $\mathbf{S I}^{3}$ (T. brucei) & 188 & 118 & 54 & 56 & $>24$ \\
\hline $\mathbf{E C}_{50}($ T. cruzi, $\mu \mathrm{M})$ & $1.85 \pm 0.29$ & $0.44 \pm 0.01$ & $1.72 \pm 0.39$ & $0.54 \pm 0.08$ & $>20$ \\
\hline $\mathbf{E C}_{90}$ (T. cruzi, $\left.\mu \mathrm{M}\right)$ & $3.41 \pm 0.14$ & $1.29 \pm 0.05$ & $2.98 \pm 0.19$ & $1.00 \pm 0.04$ & $>20$ \\
\hline SI (T. cruzi) & 18 & 10 & 5.6 & 7.6 & - \\
\hline
\end{tabular}

${ }^{1} \mathrm{EC}_{50}$ : effective concentration to reduce parasite growth by $50 \% ;{ }^{2} \mathrm{EC}_{90}$ : effective concentration to reduce parasite growth by $90 \%{ }^{3}$ SI: Selectivity index; ratio of mammalian cell and parasite $\mathrm{EC}_{50}$ values.

\subsection{Molecular Properties of Compounds $\mathbf{1}$}

A number of molecular properties are usually considered at early stages of the drug discovery process for the prediction of some important pharmacokinetic attributes. High oral bioavailability is a desirable feature for most drug candidates. According to the widely known Lipinski's rules, good oral bioavailability can be expected for compounds with molecular weight below 500 daltons, calculated $\log \mathrm{P}$ (as a measure of lipophilicity) below 5 , and no more than five hydrogen bond donors 
and ten hydrogen bond acceptors [15]. Additionally, other molecular properties have been reported to influence oral bioavailability, and hence are also commonly taken into account in early-phase drug discovery. Thus, low molecular flexibility-measured by the number of rotatable bonds, with a cutoff value of 10, and low polar surface area, measured as the sum of surface contributions of polar atoms or as the sum of tabulated surface contributions of polar fragments (topological polar surface area or TPSA) [16], with a cutoff value of $140 \AA^{2}$-are also delimiters of good oral bioavailability [17].

Brain penetration is also a desirable pharmacokinetic attribute for antitrypanosomal agents, purported to be effective in the late stage of sleeping sickness, when the T. brucei parasites invade the central nervous system (CNS). Brain permeation is usually determined experimentally through the well-known in vitro parallel artificial membrane permeability assay for blood-brain barrier (PAMPA-BBB) [18]. Of note, researchers from Pfizer recently disclosed an in silico method for the evaluation of the likeliness of a given compound to act in the CNS [19], which is based on the calculation of the so-called CNS multiparameter optimization (CNS MPO) algorithm [20,21]. This algorithm can be rapidly calculated from several of the above-mentioned molecular properties-namely, molecular weight, calculated $\log \mathrm{P}$, hydrogen bond donors, and TPSA, together with calculated $\log \mathrm{D}$ and $\mathrm{pK}_{\mathrm{a}}$ of the most basic center [20]. Compounds with CNS MPO desirability scores over 4 (in a scale from 0 to 6) tend to display favorable pharmacokinetic and safety attributes for CNS drugs.

To get insight into the potential oral bioavailability and brain permeability of the target compounds, we experimentally determined their PAMPA-BBB permeabilities through the method of Di et al. [18] and calculated the molecular properties that influence oral bioavailability and the CNS MPO scores, using the Molinspiration calculation software [22], the ChemAxon Marvin Suite 17.28 (for clog D (at pH 7.4) and pKa values), and a Pfizer's Excel active table, which is available online [20] (Table 3). With regard to the experimentally determined PAMPA-BBB permeabilities (Pe), according to the limits established by Di et al. [18], compound 1e is the only one with low BBB permeation $(\mathrm{Pe}<1.89)$, probably as a consequence of its zwitterionic character at the $\mathrm{pH}$ of the assay, which would preclude its permeation by passive diffusion, whereas the Pe values of the rest of compounds are in the range of uncertain permeability $\left(5.16>\operatorname{Pe~} 10^{-6} \mathrm{~cm} \mathrm{~s}^{-1}>1.89\right)$. However, Pe values of compounds 1a and $\mathbf{1 b}$ are interestingly close to the threshold for high BBB permeability (Pe > 5.16). Interestingly, CNS MPO scores close to the threshold for a full alignment of pharmacokinetic properties were found for most of our compounds (see Table 3 and Supplementary Information, SI), which further supports their usefulness in late-stage sleeping sickness. Moreover, we found that these compounds are compliant with Lipinski's rules, and have TPSA values and a number of rotatable bonds (nrotb) that are favorable for a good oral bioavailability (Table 3).

Table 3. Molecular properties of compounds 1.

\begin{tabular}{cccccc}
\hline & $\mathbf{1 a}$ & $\mathbf{1 b}$ & $\mathbf{1 c}$ & $\mathbf{1 d}$ & $\mathbf{1 e}$ \\
\hline PAMPA-BBB (Pe) & 4.2 & 4.9 & 2.6 & 2.4 & 1.6 \\
CNS MPO & 3.3 & 3.5 & 3.7 & 1.4 & 5.8 \\
miLogP & 3.30 & 3.93 & 4.04 & 5.67 & -1.35 \\
MW & 381.54 & 461.58 & 446.01 & 486.04 & 382.46 \\
nOHNH & 0 & 0 & 0 & 0 & 0 \\
nON & 2 & 4 & 3 & 2 & 4 \\
nviolations & 0 & 0 & 0 & 1 & 0 \\
TPSA & 8.82 & 27.29 & 18.05 & 8.82 & 48.95 \\
nrotb & 5 & 7 & 6 & 5 & 6 \\
\hline
\end{tabular}

CNS MPO: CNS multiparameter optimization; MW: molecular weight; nOHNH: number of hydrogen bond donors; nON: number of hydrogen bond acceptors; nrotb: number of rotatable bonds; nviolations: number of violations of Lipinski's rules; PAMPA-BBB (Pe): parallel artificial membrane permeability assay for blood-brain barrier permeability; miLogP: LogP calculated using Molinspiration; TPSA: topological polar surface area. 


\section{Materials and Methods}

\subsection{Compound Preparation General Methods}

$N$-(aryl)propargylamines 2 were prepared from the corresponding amines, aldehydes, and terminal alkynes by a modification of Li and Wei's protocol [12]: In a Schlenk tube, $2.0 \mathrm{mmol}$ (1.00 equiv.) of the aldehyde and $2.2 \mathrm{mmol}$ (1.1 equiv.) of the amine were dissolved in $5 \mathrm{~mL}$ of THF under $\mathrm{N}_{2}$ atmosphere. The tube was then sealed and the mixture was heated at $60{ }^{\circ} \mathrm{C}$ until complete consumption of the aldehyde $(\approx 2 \mathrm{~h})$. Next, $\mathrm{CuBr}(30 \mathrm{~mol} \%), \mathrm{RuCl}_{3}(3 \mathrm{~mol} \%)$, and the alkyne ( $2.4 \mathrm{mmol}, 1.2$ equiv.) were added. The mixture was stirred at room temperature $(\approx 30 \mathrm{~min})$, and then heated at $50{ }^{\circ} \mathrm{C}$ until complete consumption of the imine $(\approx 24 \mathrm{~h})$. Afterwards, the solution was cooled, poured into water $(40 \mathrm{~mL})$, and extracted with DCM $(3 \times 20 \mathrm{~mL})$. The combined organic phases were washed with water $(2 \times 20 \mathrm{~mL})$, dried $\left(\mathrm{anh} . \mathrm{MgSO}_{4}\right)$, and concentrated under vacuum. Purification by flash chromatography on silica gel (hexanes/AcOEt) afforded the corresponding propargylamines.

Tetrasubstituted imidazolium salts $\mathbf{1}$ were prepared from the corresponding propargylamines and isocyanides through the recent procedure reported by the group [11]: Propargylamine 2 (0.5 mmol, 1 equiv.) was dissolved in THF $(1.5 \mathrm{~mL})$ and $\mathrm{ACN}(1.5 \mathrm{~mL})$ under $\mathrm{N}_{2}$ atmosphere. Next, the isocyanide ( $0.5 \mathrm{mmol}, 1$ equiv.) was added. A $4 \mathrm{M} \mathrm{HCl}$ solution in dioxane ( $125 \mu \mathrm{L}, 1$ equiv.) was added and the mixture stirred at room temperature until total consumption of the propargylamine $(\approx 4 \mathrm{~h})$. Next the reaction was quenched with saturated $\mathrm{Na}_{2} \mathrm{CO}_{3}$ aqueous solution $(20 \mathrm{~mL})$ and extracted with $\mathrm{AcOEt}$ $(3 \times 10 \mathrm{~mL})$. The combined organic phases were dried $\left(\mathrm{anh} . \mathrm{MgSO}_{4}\right)$ and concentrated under vacuum to afford the corresponding imidazolium salts 1 . Analytically pure samples of the products were obtained by flash chromatography on silica gel (hexanes/EtOH 9:1 to 7:3 v/v).

For detailed protocols and compound characterization, see Reference [11] and the Supporting Information.

\subsection{Biological Activity Determination}

\subsubsection{T. brucei Culturing and Evaluation of Trypanocidal Activity}

Bloodstream form T. brucei (strain 221) were grown as described previously [23]. Compound activity was determined by culturing parasites in the presence of different concentrations so that the levels which inhibited growth by $50 \%\left(\mathrm{EC}_{50}\right)$ and $90 \%\left(\mathrm{EC}_{90}\right)$ could be calculated. Logarithmically growing T. brucei were diluted to $2.5 \times 10^{4} \mathrm{~mL}^{-1}$ and aliquoted into 96 -well plates. Then, compounds were added at a range of concentrations and the plates incubated at $37^{\circ} \mathrm{C}$. Each compound was tested in triplicate. Resazurin was added after $48 \mathrm{~h}$ and the plates incubated for a further $16 \mathrm{~h}$. The plates were then read in a Spectramax plate reader. Results were analyzed using GraphPad Prism.

\subsubsection{T. cruzi Culturing and Evaluation of Trypanocidal Activity}

T. cruzi epimastigotes (CL Brener stain) were cultured at $28{ }^{\circ} \mathrm{C}$ [24]. Activity was determined using 96-well plate assays. Cultures were diluted to $2.5 \times 10^{5} \mathrm{~mL}^{-1}$, aliquoted in triplicate at a range of compound concentrations, and incubated for 4 days. Resazurin was added and the plates incubated for a further 3 days, then read in a Spectramax plate reader as outlined above.

\subsubsection{Cytotoxic Activity against Rat Skeletal Myoblast L6 Cells}

Cytotoxicity was assessed using microtiter plates following a previously described protocol [25]. Briefly, L6 cells were seeded at $1 \times 10^{4} \mathrm{~mL}^{-1}$ in $200 \mu \mathrm{L}$ of growth medium at different compound concentrations. The plates were incubated for 6 days at $37^{\circ} \mathrm{C}$ and then $20 \mu \mathrm{L}$ resazurin was added to each well. After a further $8 \mathrm{~h}$ incubation, fluorescence was determined using a Spectramax plate reader. 


\subsection{Cheminformatics Calculations}

The Molinspiration package [22] was used to calculate the molecular properties (miLog P, topological polar surface area (TPSA), molecular weight (MW), number of hydrogen bond acceptors (nON), number of hydrogen bond donors ( $\mathrm{nOHNH}$, number of rotatable bonds (nrotb)), and number of violations of Lipinski's rules (nviolations). The CNS MPO scores were calculated according to Reference [20]. For the full data, see the Supplementary Materials.

\subsection{Determination of Brain Permeability: PAMPA-BBB Assay}

The in vitro permeability (Pe) of the tested compounds and fourteen reference drugs through a lipid extract of porcine brain membrane was assessed by using a parallel artificial membrane permeation assay. Commercial drugs and the target compounds were tested using a mixture of phosphate-buffered saline (PBS): EtOH 70:30. The assay was validated by comparing the experimental permeability with the reported values of the reference drugs. A lineal correlation between experimental and reported permeability values of the fourteen commercial drugs was established (experimental $\mathrm{Pe}=1.6374 \times$ reported $\left.\mathrm{Pe}-1.3839 ; \mathrm{R}^{2}=0.9203\right)$. From this equation and the limits established by $\mathrm{Di}$ et al. [18] for $\mathrm{BBB}$ permeation, three ranges of permeability were considered: compounds of high BBB permeation (CNS+): Pe $\left(10^{-6} \mathrm{~cm} \mathrm{~s}^{-1}\right)>5.16$; compounds of low BBB permeation (CNS-): Pe $\left(10^{-6} \mathrm{~cm} \mathrm{~s}^{-1}\right)<1.89$; and compounds of uncertain BBB permeation (CNS \pm ): $5.16>$ $\operatorname{Pe}\left(10^{-6} \mathrm{~cm} \mathrm{~s}^{-1}\right)>1.89$.

\section{Conclusions}

In conclusion, we have demonstrated that complex tetrasubstituted imidazolium salts are active antiparasitic agents against both African and American trypanosomiases. The remarkably high potency, good selectivity indexes, and favorable physicochemical/pharmacokinetic properties of these compounds make them promising hits for future studies. These properties can be further optimized by exploiting the several diversity points of the scaffold and its multicomponent origin. Moreover, the synthetic approach described here will facilitate the generation of new compounds and the assessment of structure-activity relationship SAR due to its modular nature.

Supplementary Materials: The following file is available online: a pdf file containing the experimental procedures for the synthesis of the compounds, their chemical characterization, additional data on the calculations, PAMPA and biological activity determinations.

Acknowledgments: We acknowledge support from DGICYT-Spain (CTQ-2015-67870P, SAF2014-57094-R), and the Generalitat de Catalunya (2014 SGR 52, 137 and 1189). J.M.K. acknowledges support from the British Heart Foundation.

Author Contributions: O.G. and R.L. conceived and designed the experiments; O.G., N.K. and M.R. performed the chemical experiments; M.C.T. and J.M.K. did the biological testing; O.D.P., D.M.-T. and B.P. calculated the physicochemical profiles and the PAMPA scores. All authors analyzed the data and wrote the paper.

Conflicts of Interest: The authors declare no conflict of interest.

\section{References}

1. Word Health Organization Media Centre. Trypanosomiasis, Human African (Sleeping Sickness). Available online: http:/ / www.who.int/mediacentre/factsheets/fs259/en/ (accessed on 15 November 2017).

2. Word Health Organization Media Centre. Chagas Disease (American Trypanosomiasis). Available online: http:/ / www.who.int/mediacentre/factsheets/fs340/en/ (accessed on 15 November 2017).

3. Njoroge, M.; Njuguna, N.M.; Mutai, P.; Ongarora, D.S.B.; Smith, P.W.; Chibale, K. Recent approaches to chemical discovery and development against malaria and the neglected tropical diseases human African trypanosomiasis and schistosomiasis. Chem. Rev. 2014, 114, 11138-11163. [CrossRef] [PubMed]

4. Bolognesi, M.L. Multi-target-directed ligands as innovative tools to combat trypanosomatid diseases. Curr. Top. Med. Chem. 2011, 11, 2824-2833. [CrossRef] [PubMed] 
5. Mäser, P.; Wittlin, S.; Rottmann, M.; Wenzler, T.; Kaiser, M.; Brun, R. Antiparasitic agents: New drugs on the horizon. Curr. Opin. Pharmacol. 2012, 12, 562-566. [CrossRef] [PubMed]

6. Field, M.C.; Horn, D.; Fairlamb, A.H.; Ferguson, M.A.; Gray, D.W.; Read, K.D.; De Rycker, M.; Torrie, L.S.; Wyatt, P.G.; Wyllie, S.; et al. Anti-trypanosomatid drug discovery: An ongoing challenge and a continuing need. Nat. Rev. Microbiol. 2017, 15, 217-231. [CrossRef] [PubMed]

7. Centers for Disease Control and Prevention. Neglected Tropical Diseases. Available online: http:/ / www. cdc.gov/globalhealth/ntd/ (accessed on 20 November 2017).

8. Gravel, J.; Schmitzer, A.R. Imidazolium and benzimidazolium-containing compounds: From simple toxic salts to highly bioactive drugs. Org. Biomol. Chem. 2017, 15, 1051-1071. [CrossRef] [PubMed]

9. Faral-Tello, P.; Liang, M.; Mahler, G.; Wipf, P.; Robello, C. Imidazolium compounds are active against all stages of Trypanosoma cruzi. Int. J. Antimicrob. Agents 2014, 43, 262-268. [CrossRef] [PubMed]

10. Kishore, K.G.; Ghashghaei, O.; Estarellas, C.; Mestre, M.M.; Monturiol, C.; Kielland, N.; Kelly, J.M.; Francisco, A.F.; Jayawardhana, S.; Muñoz-Torrero, D.; et al. Insertion of isocyanides into N-Si bonds: Multicomponent reactions with azines leading to potent antiparasitic compounds. Angew. Chem. Int. Ed. 2016, 55, 8994-8998. [CrossRef] [PubMed]

11. Ghashghaei, O.; Revés, M.; Kielland, N.; Lavilla, R. Modular access to tetrasubstituted imidazolium salts through acid-catalyzed addition of isocyanides to propargylamines. Eur. J. Org. Chem. 2015, 4383-4388. [CrossRef]

12. Li, C.-J.; Wei, C. Highly efficient Grignard-type imine additions via C-H activation in water and under solvent-free conditions. Chem. Commun. 2002, 268-269. [CrossRef]

13. Tong, S.; Wang, Q.; Wang, M.-X.; Zhu, J. Tuning the reactivity of isocyano group: Synthesis of imidazoles and imidazoliums from propargylamines and isonitriles in the presence of multiple catalysts. Angew. Chem. Int. Ed. 2015, 54, 1293-1297. [CrossRef] [PubMed]

14. Wilkinson, S.R.; Taylor, M.C.; Horn, D.; Kelly, J.M.; Cheeseman, I. A mechanism for cross-resistance to nifurtimox and benznidazole in trypanosomes. Proc. Natl. Acad. Sci. USA 2008, 105, 5022-5027. [CrossRef] [PubMed]

15. Lipinski, C.A.; Lombardo, F.; Dominy, B.W.; Feeney, P.J. Experimental and computational approaches to estimate solubility and permeability in drug discovery and development settings. Adv. Drug Deliv. Rev. 1997, 23, 4-25. [CrossRef]

16. Ertl, P.; Rohde, B.; Selzer, P. Fast calculation of molecular polar surface area as a sum of fragment-based contributions and its application to the prediction of drug transport properties. J. Med. Chem. 2000, 43, 3714-3717. [CrossRef] [PubMed]

17. Veber, D.F.; Johnson, S.R.; Cheng, H.-Y.; Smith, B.R.; Ward, K.W.; Kopple, K.D. Molecular properties that influence the oral bioavailability of drug candidates. J. Med. Chem. 2002, 45, 2615-2623. [CrossRef] [PubMed]

18. Di, L.; Kerns, E.H.; Fan, K.; McConnell, O.J.; Carter, G.T. High throughput artificial membrane permeability assay for blood-brain barrier. Eur. J. Med. Chem. 2003, 38, 223-232. [CrossRef]

19. Viswanadhan, V.N.; Balan, C.; Hulme, C.; Cheetham, J.C.; Sun, Y. Knowledge-based approaches in the design and selection of compound libraries for drug discovery. Curr. Opin. Drug Discov. Dev. 2002, 5, 400-406. [PubMed]

20. Wager, T.T.; Hou, X.; Verhoest, P.R.; Villalobos, A. Moving beyond rules: The development of a central nervous system multiparameter optimization (CNS MPO) approach to enable alignment of druglike properties. ACS Chem. Neurosci. 2010, 1, 435-449. [CrossRef] [PubMed]

21. Wager, T.T.; Hou, X.; Verhoest, P.R.; Villalobos, A. Central nervous system multiparameter optimization desirability: Application in drug discovery. ACS Chem. Neurosci. 2016, 7, 767-775. [CrossRef] [PubMed]

22. Calculations were Performed Using the Molinspiration Package. Available online: http://molinspiration. com (accessed on 11 December 2017).

23. Wilkinson, S.R.; Prathalingam, S.R.; Taylor, M.C.; Ahmed, A.; Horn, D.; Kelly, J.M. Functional characterisation of the iron superoxide dismutase gene repertoire in Trypanosoma brucei. Free Radic. Biol. Med. 2006, 40, 198-209. [CrossRef] [PubMed]

24. Kendall, G.; Wilderspin, A.F.; Ashall, F.; Miles, M.A.; Kelly, J.M. Trypanosoma cruzi glycosomal glyceraldehyde-3-phosphate dehydrogenase does not conform to the "hotspot" topogenic signal model. EMBO J. 1990, 9, 2751-2758. [PubMed] 
25. Bot, C.; Hall, B.S.; Bashir, N.; Taylor, M.C.; Helsby, N.A.; Wilkinson, S.R. Trypanocidal activity of aziridinyl nitrobenzamide prodrugs. Antimicrob. Agents Chemother. 2010, 54, 4246-4252. [CrossRef] [PubMed]

Sample Availability: Samples of the compounds 1a-e are not available from the authors. article distributed under the terms and conditions of the Creative Commons Attribution (CC BY) license (http:/ / creativecommons.org/licenses/by/4.0/). 